\title{
Frequency of arrhythmias and other cardiac abnormalities in fulminant hepatic failure
}

\author{
M. J. Weston, I. C. Talbot, P. J. N. Howorth, A. K. Mant, R. Capildeo, and Roger Williams \\ From the Liver Unit, Departments of Chemical Pathology and Morbid Anatomy, King's College Hospital \\ and Medical School, London
}

In a series of 106 patients with fulminant hepatic failure and grade 4 encephalopathy, cardiac arrhythmias and other abnormalities occurred in 92 per cent. The most common was sinus tachycardia (75\%) and this was the only abnormality in 22 per cent of the patients. Sudden cardiac arrest occurred in 25 per cent, various ectopic beats in 20 per cent, and heart block or bradycardia in 18 per cent. Other electrocardiographic abnormalities, mostly of the $T$ wave and $S T$ segment, were found in 31 per cent. Cardiac and respiratory arrests were usually unrelated to each other and both frequently occurred without warning. Only 7 sut of 71 patients with arrhythmias other than sinus tachycardia survived, compared with 15 out of 31 patients without them $(P<0.005)$. During the latter part of the series when an arrhythmia computer was used to monitor 38 patients, it was shown that significantly lower arterial oxygen levels occurred in those with arrhythmias, other than sinus tachycardia, than in those without. They were also found to be more acidotic and hyperkalaemic, and a higher number required dialysis and ventilation. Macroscopical cardiac abnormalities including scattered petechial haemorrhages, small pericardial effusions, and fatty, pale, and flabby ventricles, were found at necropsy in 64 per cent of the patients examined. Combinations of these macroscopical abnormalities occurred, particularly in the paracetamol overdose group. Another necropsy finding of possible significance in the pathogenesis of arrhythmias was cerebral oedema, present in 48 per cent of the patients examined, and often associated with coning of the brain stem. However, 7 of the 16 patients who suffered asystolic cardiac arrests had no macroscopical abnormality of either heart or brain.

In the management of patients with fulminant hepatic failure continuous cardiac monitoring is essential. Correction of the biochemical and coagulation defects may decrease the frequency of arrhythmias but studies of the mechanism and control of cerebral oedema and its relation to cardiovascular function are urgently needed.

Fulminant hepatic failure impairs the function of many organs other than the liver. In the cardiovascular system, various changes including peripheral vasodilatation, tachycardia, high cardiac 'output, and hypotension have been described (Trey and Davidson, 1970; Williams, 1974). They have been little studied, though experimental interest in the effects of bile on the cardiovascular system dates from the turn of the century (Meltzer and Salant, 1905; Bunting and Brown, 1911; Emerson, 1929; Wakim, Essex, and Mann, 1940). Abnormalities of the $T$ wave in the electrocardiogram of patients with infectious hepatitis, all of whom survived, were described by Dehn, Feil, and Received 8 December 1975.
Rinderknecht (1946) and in 4 patients who died from viral hepatitis histological changes suggesting myocarditis were found by Saphir, Amromin, and Yokoo (1956). Bell (1971) drew attention to widespread petechial haemorrhages, infiltrations of lymphocytes, fatty degeneration, flabby dilated ventricles, and oedema of the subendocardial tissue found at necropsy in patients dying of viral hepatitis. Many of these patients had suffered cardiac arrhythmias during their illness.

Apart from direct myocardial involvement by hepatitis $A$ and $B$ viruses, many of the biochemical abnormalities, as well as changes in brain-stem function, present in fulminant hepatic failure could be important in the pathogenesis of these arrhyth- 
mias. These factors are considered in this study and an analysis is made of the frequency and types of arrhythmia occurring in 106 patients with fulminant hepatic failure. In the latter part of the series, a cardiac arrhythmia computer was used to try to identify specific patterns or sequences of arrhythmias to help understand their development and prevention.

\section{Patients and methods}

The 106 patients were admitted to the Liver Unit, King's College Hospital, during the period October 1969 to October 1974. All of them deteriorated to grade 4 encephalopathy (as defined by Trey and Davidson, 1970) during the course of their illness. The liver failure was most commonly caused by hepatitis A or B or by overdoses of paracetamol taken in suicidal attempts (Table 1).

The patients all received standard supportive therapy with intensive nursing care and 24-hour medical cover, and over the latter 12 months of the study were looked after in a purpose-designed, two-bedded liver failure unit. Of the earlier patients, 6 were treated with exchange transfusion, 4 with extracorporeal liver perfusion, and 1 was crosscirculated with a human volunteer. In the 34 cases investigated since October 1973, a new technique of temporary liver support based on charcoal haemoperfusion was used (Gazzard et al., 1974). Serum electrolyte estimations, blood gas analysis, liver function tests, and coagulation studies were carried out daily in all patients, or more frequently if indicated.

Necropsies were carried out in 76 of the 84 patients who did not recover. Particular attention was paid to the presence of macroscopical cardiac abnormalities, and in 24 patients sections of the

TABLE 1 Aetiology of fulminant hepatic failure in 106 patients together with mortality

\begin{tabular}{lcc}
\hline Aetiology & No. of cases & $\%$ Mortality \\
\hline Virus hepatitis & & \\
Type A & 19 & 73 \\
$\quad$ Type B & 16 & 81 \\
Unclassified & 9 & 100 \\
Paracetamol overdose & 43 & 77 \\
Halothane associated & 12 & 92 \\
Other (drug reactions, etc.) & 7 & 43 \\
\hline Total & 106 & 79 \\
\hline
\end{tabular}

Note: In virus hepatitis type $\mathrm{B}, \mathrm{HBsAg}$ was detected by immunodiffusion or radioimmunoassay. In the unclassified cases sera had not been tested. left ventricle were stained for microscopy ( 16 with haematoxylin and eosin and 8 with Oil Red O). The brain was also routinely examined for cerebral oedema and evidence of coning.

Information on arrhythmias in the first 68 patients was obtained retrospectively from notes, electrocardiograms, and rhythm strips where possible. In 38 of the more recent cases continuous cardiac monitoring was carried out with equipment supplied by Messrs Simonsen and Weel which comprised an arrhythmia recorder, single channel recorder, computer, memory twinscope, and servograph pen drive for arrhythmia write-out. When a steady trace had been produced, three consecutive complexes were compared with respect to the $R R$ interval, $Q R S$ width, and QRST area. If any of these differed by \pm 20 per cent or more of the value stored in the memory, it was registered on the recording system which presented arrhythmias in the form of a histogram.

\section{Results}

Only 9 of the 106 patients had no record of an arrhythmia while in grade 4 encephalopathy. Sinus tachycardia (greater than 100 beats a minute) was the commonest of the arrhythmias, being present in 79 per cent of the patients. It was the only abnormality in 24 per cent. It often occurred before deep coma, and in many instances persisted throughout the illness. Cardiac arrest took place in 27 per cent of the patients. These were asystolic in 16 patients with the remainder caused by ventricular fibrillation or an unspecified cause (retrospective survey). Eleven patients suffered from atrial fibrillation and 8 from supraventricular tachycardia. Ventricular ectopics occurred in 8 patients and atrial or nodal ectopics in 6 . Four had more than one type of ectopic, and in another 3 the nature of the ectopic beat was not specified. Ventricular tachycardia was observed in 5 patients and an idioventricular rhythm in 1 . Sinus bradycardia was observed in 5 patients and bradycardia of an unspecified nature in another 9. Complete heart block was seen in 2 patients and 2 other patients had first or second degree heart block. Abnormalities of the ST segment and T wave in the electrocardiogram were frequently seen but did not appear to relate to any specific arrhythmia. It was not possible to correlate either the type or frequency of arrhythmia with the aetiology of the fulminant hepatic failure.

Usually the arrhythmias were of relatively short duration and lasted for only a few hours at the most. They either reverted spontaneously or responded 


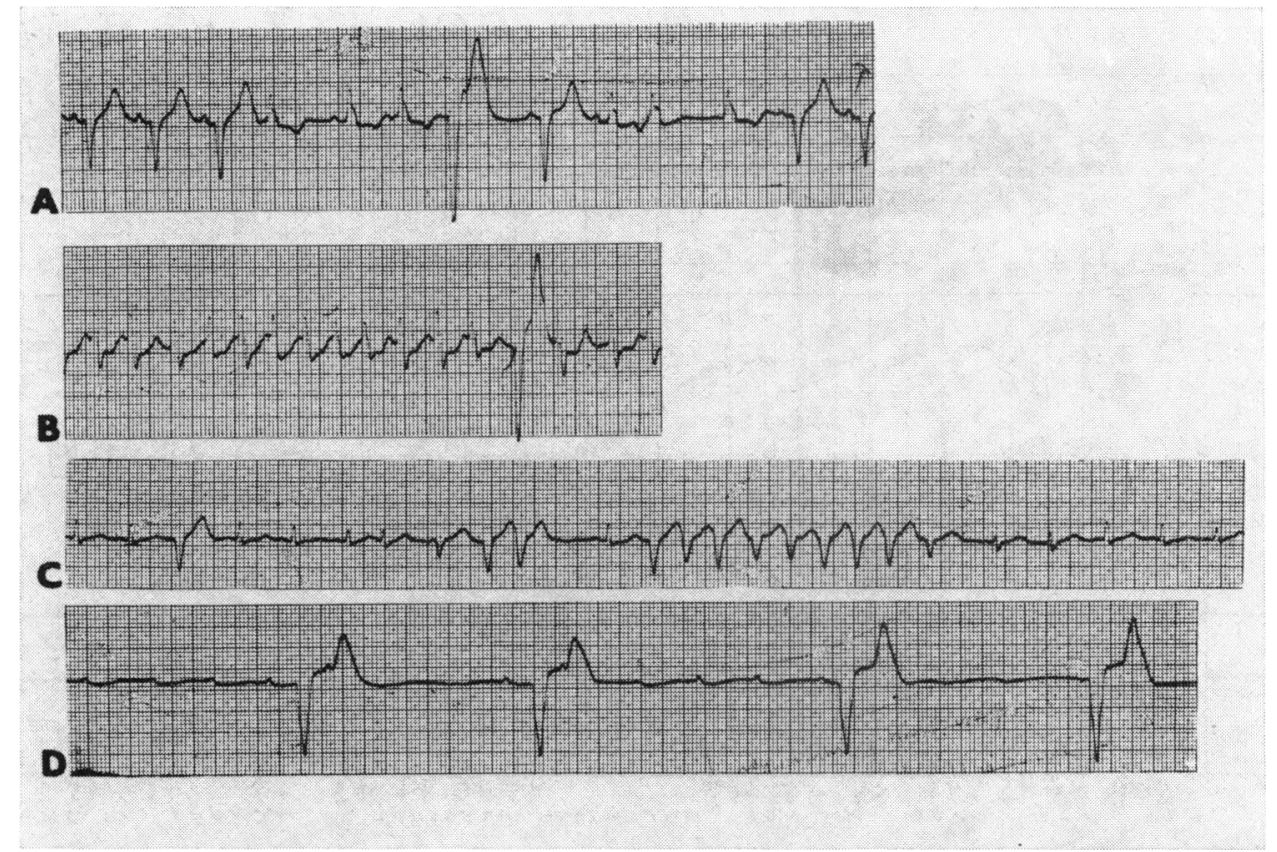

FIG. 1 Rhythm strips obtained from a 69-year-old man while in grade 4 coma from fulminant hepatic failure caused by a presumed type $A$ hepatitis: $(A)$ Sinus rhythm, intermittent bundlebranch block, and ventricular ectopics; $(B)$ atrial fibrillation which reverted to sinus rhythm with direct current version; (C) episode of ventricular tachycardia; (D) complete heart block. At necropsy the myocardium appeared normal though there were numerous petechial haemorrhages in the pericardium. There was no evidence of brain-stem coning.

to standard measures (for example, digoxin for supraventricular tachycardia which occurred in 10 patients, atropine for bradycardia, direct current shock for ventricular tachycardia). In addition to the 24 per cent who had sinus tachycardia alone another 6 patients had single types of arrhythmia only ( 2 had asystolic cardiac arrests and one each with complete heart block, Wenckebach phenomenon, paroxysmal ventricular tachycardia, and supraventricular tachycardia). Another 2 had $T$ wave abnormalities alone. The remaining 65 patients had more than one type of arrhythmia and many had several (Fig. 1), but apart from usually being preceded by a sinus tachycardia, there was no specific pattern in their evolution. Their occurrence was not related to the duration of coma and one patient with a paracetamol overdose sustained an asystolic cardiac arrest while still rousable. Though some of the older patients were troubled by more persistent arrhythmias, their frequency did not appear to increase with age. Thus, the average age of the 11 patients who had periods of atrial fibrillation (an arrhythmia whose incidence might be expected to increase with age) was $34 \cdot 2$ (range 18 to 69 ) years, as compared with 35.4 (range 10-78) years in those without atrial fibrillation. The appearance of arrhythmias was clearly a poor prognostic sign, as 64 of the 71 patients in whom they occurred (excluding sinus tachycardia) died, compared with only 15 out of 33 without.

Cardiac arrests were frequently asystolic and resuscitation was temporarily successful in 5 instances. However, one patient in whom the liver failure had followed a massive dose of paracetamol, suffered two asystolic arrests while deeply unconscious but eventually survived. Frequently the arrests occurred without warning, though 2 of the 6 that occurred when the cardiac arrhythmia computer was in use were associated with respiratory arrests. In all, 8 of the 38 patients monitored continuously had respiratory arrests and only one of these survived, despite assisted ventilation. Respiratory arrests, like cardiac arrests, were usually preceded by obvious respiratory difficulty comprising tachypnoea, loud coarse crepitations, occasional expectoration of fresh blood, and in- 


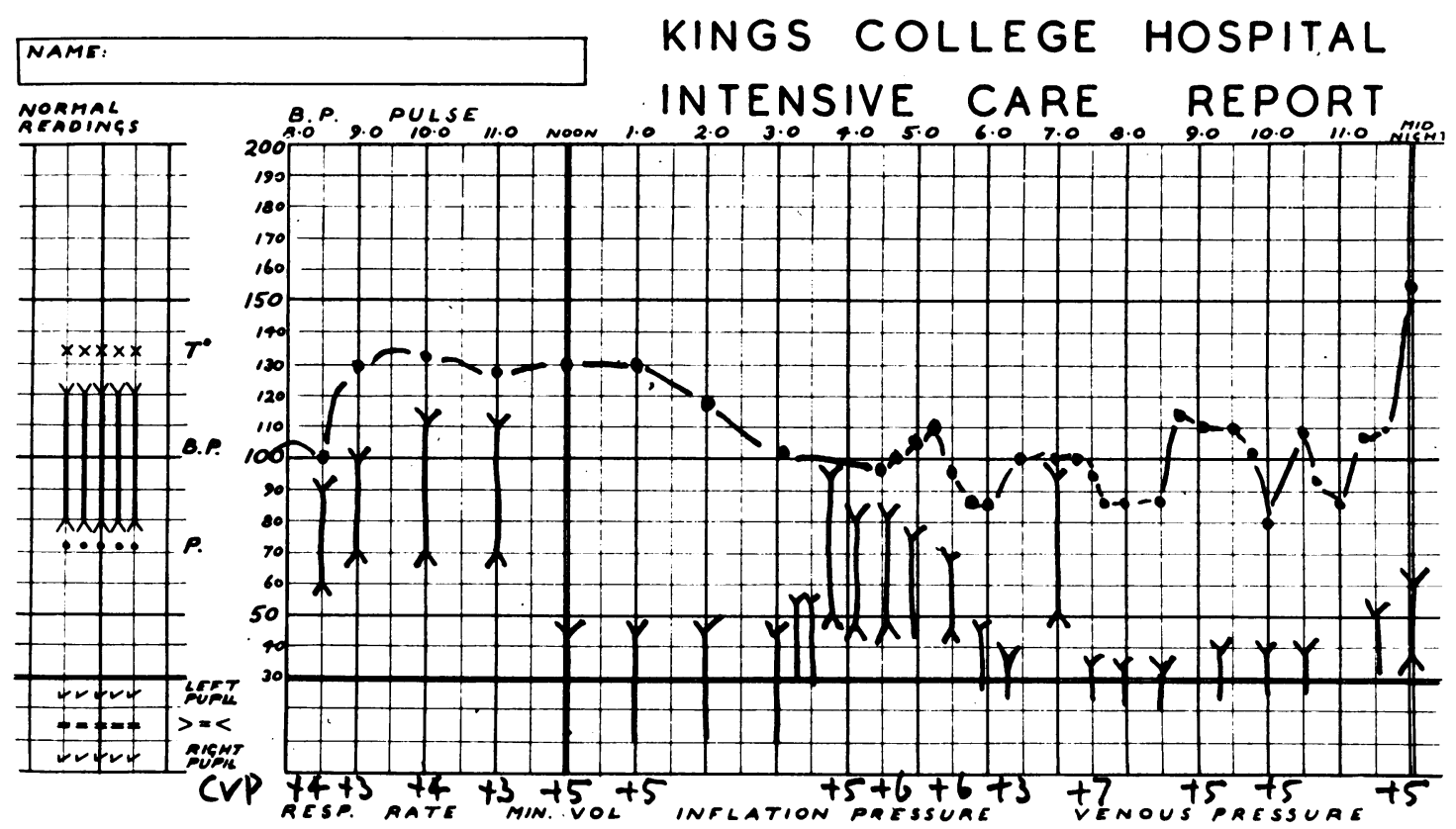

FIG. 2 Part of nursing chart showing sinus tachycardia and sudden drop of blood pressure with little change of central venous pressure, suggestive of brain-stem coning.

creasing tachycardia, though sometimes they occurred without warning. Changes of blood pressure, without clinically obvious bleeding or fluid imbalance, which we now consider indicative of brain-stem coning, were sometimes observed immediately before sudden respiratory or cardiac arrests (Fig. 2).

There were no specific arrhythmias associated with charcoal haemoperfusion though a slight fall of blood pressure (10 to $20 \mathrm{mmHg}$ ( 1.3 to 2.7 $\mathrm{kPa})$ ) often occurred within 30 minutes of the start of the procedure. In one patient with a sinus tachycardia, the pulse rate dropped by 10 per cent each time he was perfused and in a 16-year-old patient with a paracetamol overdose, pronounced $T$ wave inversion developed during one perfusion which was reversed by blood transfusion.

\section{Continuous cardiac monitoring and relevant biochemical changes in 38 patients}

Use of the cardiac arrhythmia recording apparatus itself proved satisfactory, but it was often difficult to interpret arrhythmias from the histogram alone because of artefacts arising at the patient-electrode interface. The majority of the arrhythmias recorded by the computer had also been witnessed on the oscilloscope and transcribed on a rhythm strip by the attending physician or nurse. The arrhythmia computer gave a higher recognition rate of supraventricular tachycardia, ectopic beats of all types, atrial fibrillation, and heart block than in the previous 68 patients, though the frequency of sinus tachycardia, serious ventricular arrhythmias, and cardiac arrests remained the same.

The 38 patients were divided into two groups according to the presence or absence of arrhythmias, excluding sinus tachycardia which occurred in all but 4 of them. Differences in the maximum and minimum levels of serum potassium, calcium, phosphate, and arterial hydrogen ion concentration and partial pressure of oxygen in each group (Table 2) were analysed with the Student's t-test during the period of grade 4 encephalopathy. The only significant differences found were in the mean oxygen levels which were lower $(P<0.02)$ in the patients with arrhythmias. Comparison of the maximum arterial oxygen levels showed that these were higher in the arrhythmia group $(P<0.05)$, and presumably this resulted from the more frequent oxygen enrichment and assisted ventilation requirements of this group. Though the arrhythmia group also had higher hydrogen ion concentrations, 
TABLE 2 Comparison of biochemical and blood gas analysis in 38 patients having continuous cardiac monitoring divided according to presence or absence of arrhythmias other than sinus tachycardia

\begin{tabular}{lll}
\hline & $\begin{array}{l}\text { Arrhythmias } \\
\text { Present }(n=25)\end{array}$ & Absent $(n=13)$ \\
\hline Arterial hydrogen ion concentration (nmol/1) & $53 \pm 6 \cdot 6-30 \cdot 2 \pm 1 \cdot 6$ & $38 \cdot 1 \pm 2 \cdot 1-28 \cdot 5 \pm 0 \cdot 6$ \\
Arterial oxygen levels (kPa) & $19 \cdot 6 \pm 1 \cdot 01-8 \cdot 44 \pm 0 \cdot 5$ & $15 \cdot 6 \pm 1 \cdot 5-11 \cdot 12 \pm 1 \cdot 1$ \\
Serum potassium levels (mmol/1) & $5 \cdot 34 \pm 0 \cdot 26-3 \cdot 27 \pm 0 \cdot 14$ & $4 \cdot 58 \pm 0 \cdot 19-2 \cdot 97 \pm 0 \cdot 14$ \\
Serum calcium (mmol/1) & $2 \cdot 28 \pm 0 \cdot 04-1 \cdot 93 \pm 0 \cdot 05$ & $2 \cdot 25 \pm 0 \cdot 06-1 \cdot 99 \pm 0 \cdot 05$ \\
Serum phosphorus (mmol/1) & $1 \cdot 54 \pm 0 \cdot 18-0 \cdot 57 \pm 0 \cdot 12$ & $1 \cdot 03 \pm 0 \cdot 13-0 \cdot 45 \pm 0 \cdot 09$ \\
Prothrombin time (seconds prolonged) & $23 \cdot 3 \pm 9 \cdot 43$ & $24 \cdot 1 \pm 3 \cdot 99$ \\
Plasma bilirubin $(\mu \mathrm{mol} / \mathrm{l})$ & $400 \pm 39 \cdot 3$ & $411 \cdot 9 \pm 68 \cdot 18$ \\
Survivors & 6 & 10 \\
Numbers requiring & 9 & 3 \\
$\quad$ dialysis & 13 & 2 \\
\hline ventilation & & \\
\hline
\end{tabular}

Note: The two sets of values shown for each measurement represent mean maxima and minima ( \pm 1 SEM) respectively observed while patients were in grade 4 encephalopathy. Prothrombin times and plasma bilirubin levels were the maximum recorded during this period.

potassium and phosphate levels, the differences were not statistically significant $(P>0 \cdot 2,>0 \cdot 1$, and $>0 \cdot 1$, respectively). As indices of liver cell dysfunction, the maximum levels of serum bilirubin and maximum prolongation of the one-stage prothrombin time recorded during the illness were compared, but no significant differences between the two groups of patients were found. The number of patients requiring dialysis and ventilatory support was higher in the group with arrhythmias, but this was probably only a reflection of their more severe illness and poorer prognosis already referred to.

\section{Cardiac abnormalities and other findings at necropsy}

Forty-two of the 76 hearts examined at necropsy were macroscopically abnormal (Fig. 3). In 33 hearts the main changes could be grouped as follows: a pale, flabby, fatty myocardium (11 cases), scattered petechial haemorrhages (15 cases), dilatation of the ventricles without other organic cause (7 cases), and pericardial effusions (11 cases). Ten of these cases showed more than one abnormality (9 with 2 abnormalities and one with 3). Eight of these 10 hearts came from the paracetamol overdose patients but apart from the frequency of multiple abnormalities in this group, no particular associations were found between them. Larger bleeds had occurred in the intraventricular septum in 3 of the hearts with petechial haemorrhages, and in one other case, with a diagnosis of hepatitis A infection, the myocardium was frankly haemorrhagic. Pericardial effusions were seldom more than $50 \mathrm{ml}$ in volume. They were occasionally bloodstained, but none had given rise to cardiac tamponade. Of the

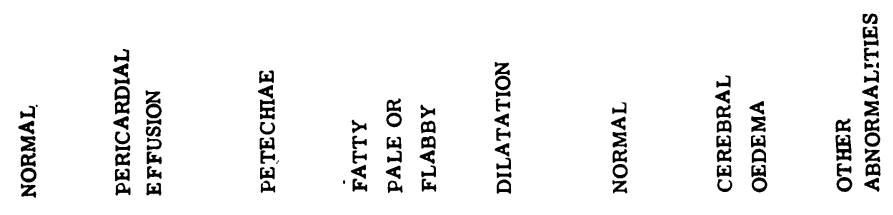

FREQUENCY OF MACROSCOPIC FINDINGS IN HEART AND B̈RAIN.

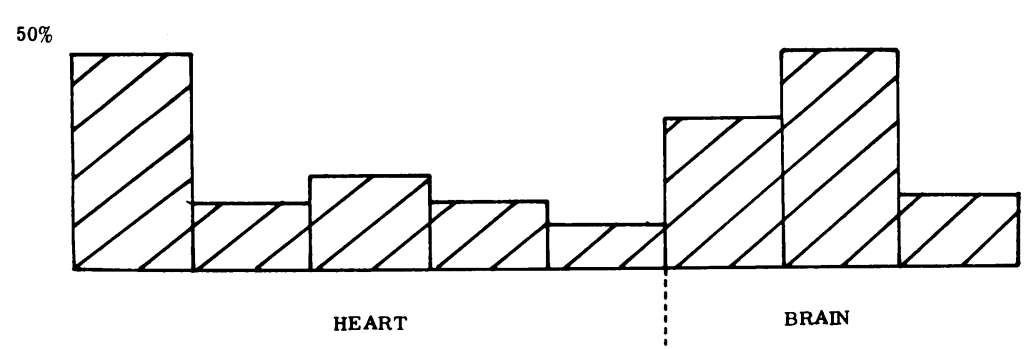

FIG. 3 Frequency of macroscopical cardiac and cerebral abnormalities at necropsy in patients dying from fulminant hepatic failure, presented in form of histograms. 
11 patients with fatty, pale, flabby hearts, 9 came from the paracetamol overdose group.

In the remaining 9 of the 42 hearts showing abnormalities, changes unrelated to hepatic failure were found. Three of these had left ventricular hypertrophy caused by longstanding hypertension and there were single cases of right ventricular hypertrophy caused by emphysema, aortic regurgitation resulting from Marfan's syndrome, a Starr valve prosthesis, and infiltration of the myocardium with excess adipose tissue. Diffuse myocardial abscesses and subacute bacterial endocarditis which had not been detected during life were found in the final two cases. They may have contracted infection as a complication of their fulminant hepatic failure.

No correlation could be detected between macroscopical abnormalities of the heart and the type of arrhythmia that had occurred during life. Indeed 3 of those with macroscopical abnormalities had no arrhythmias, 5 had had asystolic cardiac arrests, and the remaining 34 all had arrhythmias of one sort or another. On the other hand, 10 of the 34 patients with macroscopically normal hearts had cardiac arrests and the other 24 all had arrhythmias.

Paraffin sections of the left ventricle taken from 16 of the 33 patients who died from a paracetamol overdose were stained with haematoxylin and eosin and examined microscopically. Evidence of finely divided intracellular fat was found in 12 of them, irrespective of whether macroscopical changes had shown a fatty appearance. Specific staining for fat with Oil Red $O$ in another 5 showed fine intracellular droplets of fat in all of them (Fig. 4). In addition, intracellular fat was also shown with this stain in sections of the left ventricle in 2 of 3 patients who died from fulminant hepatic failure caused by viral hepatitis. In 4 cases who died from causes other than liver disease, no fat was seen with Oil Red O.

\section{Cerebral changes}

Of the 72 patients whose brains were examined at necropsy, cerebral oedema was present in 34, with structural changes caused by coning in 12 . Three of these 12 patients had suffered asystolic cardiac arrests, as had 4 others with cerebral oedema, but no coning. However, in the 9 other cases who had asystolic cardiac arrests, the brain was normal in 6 . The remaining 3 were not examined. Cerebral oedema was found in 7 of the brains examined from the 8 patients suffering respiratory arrests, with coning in 5 .

\section{Discussion}

The frequency of arrhythmias in this series was as high as 92 per cent, if sinus tachycardia is included. If the patients in whom sinus tachycardia was the only abnormality are excluded, 70 per cent had arrhythmias or other electrocardiographic abnormalities. Though a sinus tachycardia is a common finding in sick patients, the rise in pulse rate often occurred before clouding of consciousness. This clinical sign must be included together with the leucocytosis and severe prolongation of prothrombin time that may herald a fulminant course in an acute hepatitis (Ritt et al., 1969). After reviewing reports of electrocardiographic abnormalities in patients with infectious hepatitis, and from his own experience with 8 patients, Lyon (1947) concluded that the virus might directly involve the heart. However, the cardiac histology cited as evidence for this had been studied only at necropsy, and though the changes could have resulted from virus myocarditis they may possibly also have been the result of the accumulation of bilirubin, bile acids, and other potentially toxic metabolites that are inevitably retained in these cases. The diffuse fatty infiltration of the myocardial cells, which had been reported previously in patients who died from virus hepatitis, was, in our series, also found in those patients with hepatic failure from a paracetamol overdose. Reports have mentioned electrocardiographic abnormalities after paracetamol overdose (MacLean et al., 1968; Will and Tomkins, 1971; Kerr, 1973) and histological changes in the myocardium (Pimstone and Uys, 1968; Sanerkin, 1971). In one patient in this series a 12-lead electrocardiogram taken a few hours after an overdose of paracetamol (Fig. 5) when the serum level of the drug was $49 \mu \mathrm{g} / \mathrm{ml}$ showed right axis deviation and inferolateral ST and T wave changes. She developed hepatic coma and died 5 days later from an asystolic cardiac arrest. Necropsy showed mild left ventricular hypertophy, scattered petechial haemorrhages on pericardium and endocardium, and minimal atheroma of the coronary arteries. The brain was slightly swollen and congested.

To study further the possible cardiotoxic effects of this drug, we performed 12-lead electrocardiograms and serum cardiac isoenzyme estimations in 10 patients who had ingested sufficient paracetamol to sustain liver damage but who never became encephalopathic (Table 3). However, only one patient had a rise in serum creatinine phosphokinase and his electrocardiogram suggested pericarditis. In three others the $T$ waves were flattened and inverted. It is not clear whether the electrocardiographic abnormalities differed significantly from those reported by Dehn et al. (1946) in noncomatose cases of infectious hepatitis. Certainly many electrocardiographic abnormalities occurred 


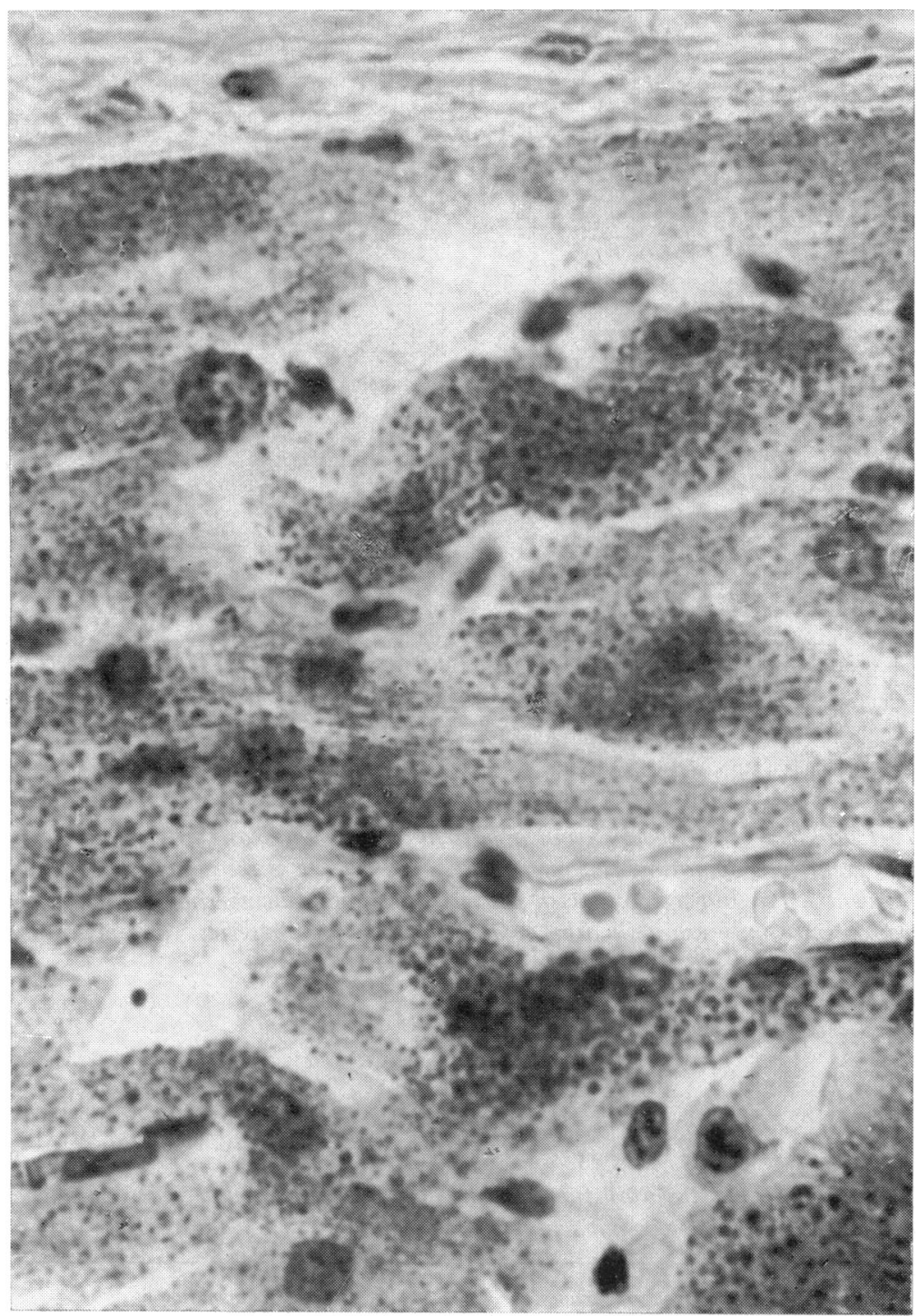

FI 4 Photomicrograph of myocardium from a patient who died after an overdose of paracetamol, showing tiny (dark) lipid droplets in the sarcoplasm. These changes were usually most prominent in the subendocardial region. Cryostat section (Oil Red $O \times 1600$ ).

in comatose patients with fulminant hepatic failure from causes other than excess paracetamol ingestion, and included widespread $\mathrm{T}$ wave inversion or ST-T segment depression, and peaked $\mathrm{T}$ waves and persistent $U$ waves when serum potassium levels were normal. The fact that multiple macroscopical abnormalities of the heart occurred more commonly in the paracetamol group may merely reflect the extremely rapid course of the liver failure in these patients.
The thrombocytopenia and hypoalbuminaemia that develop in the later stages of fulminant hepatic failure almost certainly contribute to the development of petechial haemorrhages and pericardial effusions, respectively. Many biochemical abnormalities are known to have adverse effects on myocardial function and conduction, a subject that has been reviewed in detail by Surawicz (1967). Those found in hepatic failure concern variations beyond physiological limits of serum potassium, 


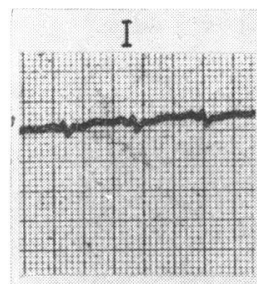

II

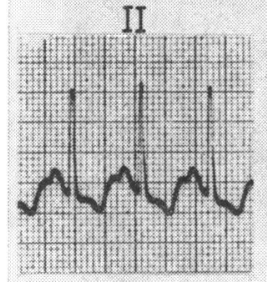

III

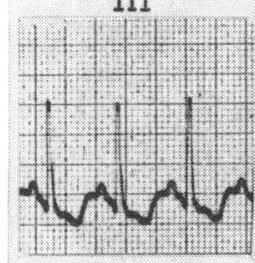

aVR

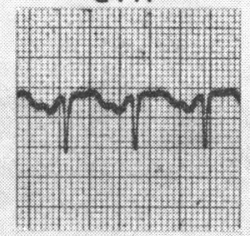

$a V L$

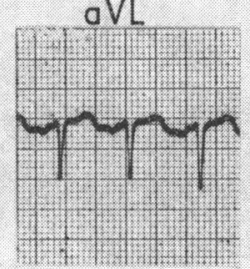

aVF

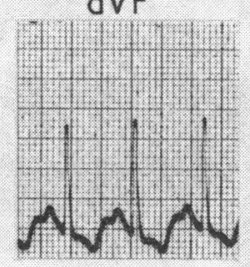

V1

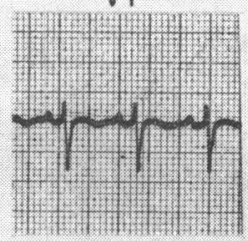

V2

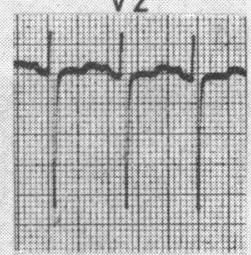

V3

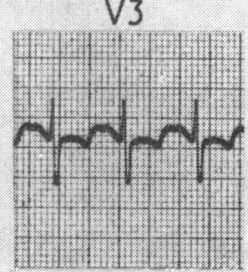

V4

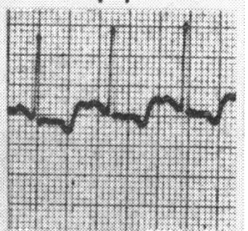

V5

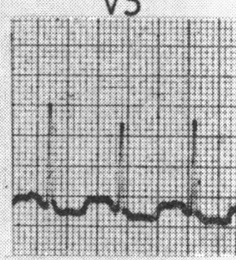

V6

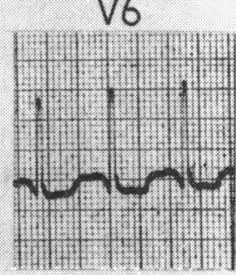

FIG. 5 12-lead electrocardiogram from a patient a few hours after a paracetamol overdose which showed right axis deviation and inferolateral $S T$ and $T$ wave changes. At necropsy there was mild left ventricular hypertrophy with scattered petechial haemorrhages on the endocardium and pericardium with only minimal atheroma of the coronary arteries. The brain was slightly swollen, and the lungs normal.

TABLE 3 Indices of cardiac and liver dysfunction in 10 patients who had ingested paracetamol in suicide attempts but in whom hepatic damage was not severe enough to lead to encephalopathy

\begin{tabular}{|c|c|c|c|c|c|c|c|}
\hline $\begin{array}{l}\text { Case } \\
\text { No. }\end{array}$ & Sex & $\begin{array}{l}\text { Age } \\
(y)\end{array}$ & $\begin{array}{l}\text { Amount of } \\
\text { paracetamol } \\
\text { ingested } \\
(\mathrm{g})\end{array}$ & Electrocardiogram & $\begin{array}{l}\text { Maximum abnorm } \\
\text { Serum creatinine } \\
\text { phosphokinase } \\
\text { (i.u.|l) }\end{array}$ & $\begin{array}{l}\text { ality of : } \\
\text { Serum bilirubin } \\
(\mu \mathrm{mol} / \mathrm{l})\end{array}$ & $\begin{array}{l}\text { Prothrombin time } \\
\text { (seconds prolonged) }\end{array}$ \\
\hline 1 & $\mathrm{~F}$ & 23 & ? & Normal & Normal & $615 \cdot 2$ & 38 \\
\hline 2 & $\mathrm{~F}$ & 33 & 25 & Inverted $T$ waves in lead 3 & - & $143 \cdot 6$ & 49 \\
\hline 3 & $\mathbf{M}$ & 24 & 50 & $\begin{array}{l}\text { Sinus bradycardia ST eleva- } \\
\text { tion V 5-6 and inferior } \\
\text { leads }\end{array}$ & 600 & $76 \cdot 9$ & 30 \\
\hline 4 & $\mathbf{M}$ & 46 & 40 & Normal & - & $138 \cdot 4$ & 19 \\
\hline 5 & $\mathbf{F}$ & 19 & 25 & $\begin{array}{l}\text { Flat } T \text { waves leads } 3 \text {, aVF, } \\
\text { 2 }\end{array}$ & Normal & $170 \cdot 9$ & 33 \\
\hline 6 & $\mathbf{M}$ & 38 & $?$ & - & Normal & $76 \cdot 9$ & 12 \\
\hline 7 & $\mathbf{F}$ & 52 & 25 & Normal & Normal & $37 \cdot 6$ & 15 \\
\hline 8 & $\mathbf{F}$ & 23 & 45 & Flat $T$ waves & Normal & $99 \cdot 1$ & 30 \\
\hline 9 & $\mathbf{M}$ & 39 & 25 & Normal & Normal & $188 \cdot 0$ & 18 \\
\hline 10 & $\mathbf{M}$ & 52 & ? & Sinus tachycardia & Normal & $73 \cdot 5$ & 17 \\
\hline
\end{tabular}


calcium, and magnesium, which all adversely affect contractility, excitability, and the conducting system. Szekeres and Papp (1967) showed that hypoxia increased the susceptibility of the heart to arrhythmias and that this is mediated by the central nervous system. Alkalosis, when induced experimentally in animals, reduces myocardial contractility and ventricular function (Cook, Webb, and Unal, 1965). The correction of cardiac arrhythmias in patients with myocardial infarction who may be suffering from a respiratory alkalosis and hypokalaemia, in addition to hypoxia, can be difficult if these abnormalities are not attended to first (Ayres and Grace, 1969). Alkalosis, comprising both a respiratory factor and hypokalaemia, is prominent early in the course of fulminant hepatic failure and before the onset of renal failure, and may require intravenous potassium supplements. Artificial ventilation sometimes provides an opportunity to correct the respiratory element, and haemodialysis the metabolic component (by lowering the $p \mathrm{H}$ of the dialysis bath). The short duration of many of the arrhythmias in our patients may account for the difficulty in implicating any one factor on specific occasions. This is why we chose to compare the maximum variations of a number of biochemical parameters during the period of grade 4 coma. The only significant differences found, namely in the arterial oxygen tensions which were both lower and higher in the group with arrhythmias, may be explained by the higher frequency of respiratory insufficiency and ventilatory support needed in this group.

The relation between lipid infiltration of the heart and sudden death has been debated for a number of years. More recently the relation of the raised blood levels of free fatty acids and arrhythmias after myocardial infarction has received clinical and experimental investigation (Kurien and Oliver, 1966; Oliver, Kurien, and Greenwood, 1968). Rowe, Neilson, and Oliver (1975) claimed reduction of the frequency of ventricular tachycardia after myocardial infarction by a nicotinic acid analogue that lowered serum-free fatty acids. Though not measured in the present series, raised serum levels of free fatty acids in fulminant hepatic failure are comparable to those occurring after myocardial infarction (Muto and Takahashi, 1964) and the accompanying hypoalbuminaemia that develops may aggravate their effects (Willebrands, Ter Welle, and Tasseron, 1973).

The influence of rises in intracranial pressure on the cardiovascular and respiratory status of these patients is difficult to assess, but could well be of major importance. Cushing (1902) first described the occurrence of cardiac and respiratory arrests in dogs with experimentally-induced rises of intracranial pressure, effects that were shown to be mediated by the vagus nerve. Moderate rises of intracranial pressure sensitize the heart to the effects of circulating catecholamines (Smith and Ray, 1971) and recently Jachuck et al. (1975) have recorded electrocardiographic abnormalities in patients occurring pari passu with changes of intracranial pressure. These comprised prominent $U$ waves, ST-T segment changes and notched T waves, and QT interval abnormalities. Many of these changes have been observed in our patients, and monitoring of intracranial pressure may prove worth while in the future.

The cause of cerebral oedema in fulminant hepatic failure, which has been reported by others (Ware, D'Agostino, and Combes, 1971), is unknown. We now suspect coning of the brain-stem in our patients whenever sudden changes of blood pressure and pulse rate occur without obvious explanation. A patient who has been quiet and unresponsive for several hours may become restless and start to groan, his circulatory and respiratory status at the same time becoming unstable. If the patient survives this episode he usually requires ventilatory support, and progressive slowing of the pulse and a drop in body temperature result over the course of several days. Though we presume in such cases that the respiratory arrest is precipitated by the rise of intracranial pressure, it is very likely that the application of ventilatory support will allow the further evolution of cerebral oedema and other changes that would not have occurred had the patient been allowed to die.

The use of the arrhythmia computer increased the rate of recognition of arrhythmias in this investigation and probably alerted clinicians to the correctable biochemical abnormalities such as hypoxia. The occurrence of cardiac arrest and hypotension almost certainly jeopardize liver and brain function further. In cases recovering spontaneously, arrhythmias disappear as the encephalopathy recedes. Though little can be done at the moment to prevent or treat cerebral oedema, or to prevent structural changes in the heart, close supervision of the patient's biochemical and respiratory status may help to avoid arrhythmias and so improve prognosis.

Continuous cardiac monitoring speeds and facilitates the identification of arrhythmias and is a valuable adjunct in their management.

This investigation is part of a programme of research into fulminant hepatic failure, receiving support from the Medical Research Council and the Wates foundation. The cardiac arrhythmia computer and other monitoring equipment was generously supplied by Messrs Simonsen and Weel Ltd. We 
are indebted to Dr. D. E. Jewitt for his help with the manuscript and to the Department of Morbid Anatomy and Chemical Pathology for their help.

\section{References}

Ayres, S. M., and Grace, W. J. (1969). Inappropriate ventilation and hypoxemia as causes of cardiac arrhythmias. American fournal of Medicine, 46, 495.

Bell, H. (1971). Cardiac manifestations of viral hepatitis. Fournal of the American Medical Association, 218, 387.

Bunting, C. H., and Brown, W. H. (1911). The pathology of intraperitoneal bile injections in the rabbit. Fournal of Experimental Medicine, 14, 445.

Cook, W. A., Webb, W. R., and Unal, M. O. (1965). Myocardial function capacity in response to compensated and uncompensated respiratory alkalosis. Surgical Forum, 16, 186.

Cushing, H. (1902). Some experimental and clinical observations concerning states of increased intracranial tension. American fournal of the Medical Sciences, 124, 375.

Dehn, H., Feil, H., and Rinderknecht, R. E. (1946). Electrocardiographic changes in cases of infectious hepatitis. American Heart fournal, 31, 183.

Emerson, W. C. (1929). The toxic constituent of the bile. Fournal of Laboratory and Clinical Medicine, 14, 635.

Gazzard, B. G., Portmann, B., Weston, M. J., Langley, P. G., Murray-Lyon, I. M., Dunlop, E. H., Flax, H., Mellon, P. J., Record, C. O., Ward, M. B., and Williams, R. (1974). Charcoal haemoperfusion in the treatment of fulminant hepatic failure. Lancet, 1, 1301.

Jachuck, S. J., Ramani, P. S., Clark, F., and Kalbag, R. M. (1975). Electrocardiographic abnormalities associated with raised intracranial pressure. British Medical fournal, 1, 242.

Kerr, D. N. S. (1973). Self-poisoning with paracetamol. Nursing Times, 69, 924.

Kurien, V. A., and Oliver, M. F. (1966). Serum-free-fattyacids after acute myocardial infarction and cerebral vascular occlusion. Lancet, $2,122$.

Lyon, E. (1947). Myocarditis in virus diseases of man. Medical Record (N.Y.), 160, 403.

MacLean, D., Peters, T. J., Brown, R. A. G., McCathie, M., Baines, G. F., and Robertson, P. G. C. (1968). Treatment of paracetamol poisoning. Lancet, 2, 849.

Meltzer, S. J., and Salant, W. (1905). Studies on the toxicity of bile: the effects of intravenous injections of bile upon the blood pressure. Fournal of Experimental Medicine, 7, 280.

Muto, Y., and Takahashi, Y. (1964). Gas chromatographic determination of plasma short chain fatty acids in diseases of the liver (in Japanese). Fournal of the Fapanese Society of Internal Medicine, 53, 838.

Oliver, M. F., Kurien, V. A., and Greenwood, T. W. (1968). Relation between serum-free-fatty-acids and arrhythmias and death after acute myocardial infarction. Lancet, 1 , 710 .

Pimstone, B. L., and Uys, C. J. (1968). Liver necrosis and myocardiopathy following paracetamol overdosage. South African Medical fournal, 42, 259.

Ritt, D. J., Whelan, G., Werner, D. J., Eigenbrodt, E. H., Schenker, S., and Combes, R. (1969). Acute hepatic necrosis with stupor or coma. Medicine, 48, 151.

Rowe, M. J., Neilson, J. M. M., and Oliver, M. F. (1975). Control of arrhythmias during myocardial infarction by antilipolytic treatment using a nicotinic-acid analogue. Lancet, 1, 295.

Sanerkin, N. G. (1971). Acute myocardial necrosis in paracetamol poisoning. British Medical fournal, 3, 478.

Saphir, O., Amromin, G. D., and Yokoo, H. (1956). Myocarditis in viral (epidemic) hepatitis. American fournal of the Medical Sciences, 231, 168.

Smith, M., and Ray, C. T. (1971). Cardiac arrhythmias, increased intracranial pressure, and the autonomic nervous system. Chest, 61, 125.

Surawicz, B. (1967). Arrhythmias and electrolyte disturbances. Bulletin of the New York Academy of Medicine, 43, 1160 .

Szekeres, L., and Papp, G. (1967). Effect of arterial hypoxia on the susceptibility to arrhythmia of the heart. Acta Physiologica Academiae Scientiarum Hungaricae, 32, 143.

Trey, C., and Davidson, C. S. (1970). Progress in Liver Disease, Vol. 3. Ed. by H. Popper and F. Schaffner. Heinemann, London.

Wakim, K. G., Essex, H. E., and Mann, F. C. (1940). The effects of whole bile and bile salts on the innervated and denervated heart. American Heart fournal, 20, 486.

Ware, A. J., D'Agostino, A. N., and Combes, B. (1971). Cerebral edema: a major complication of massive hepatic necrosis. Gastroenterology, 61, 877.

Will, E. J., and Tomkins, A. M. (1971). Acute myocardial necrosis in paracetamol poisoning. British Medical fournal, 4, 430.

Willebrands, A. F., Ter Welle, H. F., and Tasseron, S. J. A. (1973). The effect of a high molar FFA/albumin ratio in the perfusion medium on rhythm and contractility of the isolated rat heart. Fournal of Molecular and Cell Cardiology, $5,259$.

Williams, R. (1974). Fulminant viral hepatitis. Clinics in Gastroenterology, 3, 419.

Requests for reprints to Dr. Roger Williams, Liver Unit, King's College Hospital, London SE5 8RX. 